\title{
HIV-1 infected patients with suppressed plasma viremia on treatment have pro-inflammatory HDL
}

\author{
Theodoros Kelesidis ${ }^{1 *}$, Otto O Yang ${ }^{1,2}$, Judith S Currier ${ }^{1}$, Kaveh Navab ${ }^{3}$, Alan M Fogelman³, Mohamad Navab ${ }^{3}$
}

\begin{abstract}
Background: The role of pro-inflammatory lipids in systemic immune activation in HIV infection remains largely unknown. We hypothesized that HIV-1-infected persons on antiretroviral therapy would have pro-inflammatory high density lipoprotein (HDL), and that an apoA-1 mimetic peptide might reverse the inflammatory properties of $\mathrm{HDL}$ in these persons.

Methods: Plasma was obtained from $10 \mathrm{HIV}$-1-infected individuals on combination antiretroviral therapy with suppressed viremia and was incubated with the apoA-I mimetic peptide L-4F or sham-treated prior to isolation of HDL. The HDL that was isolated from each sample was tested for its ability to inhibit LDL-induced MCP-1

production in cultures of human aortic endothelial cells.

Results: We found in a small pilot study of HIV-1-infected individuals with suppressed viremia on combination antiretroviral therapy that oxidative stress and inflammation in HIV-1 are associated with a marked reduction of $\mathrm{HDL}$ antioxidant/anti-inflammatory activities. In vitro, these abnormalities were significantly improved by treatment with the apoA-1 mimetic peptide, 4F.
\end{abstract}

Conclusions: These preliminary observations suggest that the anti-inflammatory properties of $\mathrm{HDL}$ are defective in HIV-1-infected persons despite treatment that is considered to be virologically successful.

\section{Findings}

With improved antiretroviral therapies and survival among patients with HIV-1, cardiovascular disease (CVD) has become an increasingly important cause of morbidity and mortality [1]. The magnitude of the increased CVD risk among persons with HIV-1 infection is unclear, however, as are the relative contributions of viremia, immune activation, antiretroviral therapy (ART), conventional cardiovascular risk factors (such as increasing age and hypertension), and changes in systemic inflammation [1]. Understanding the relative contributions of host, virus, and antiretroviral therapy to risk of CVD in HIV-1 infection will aid in the development of strategies for prevention and treatment.

Inflammation has increasingly been recognized to be pivotal in the initiation and perpetuation of arterial injury leading to atherosclerosis and its complications [2]. Pro-inflammatory high density lipoprotein (HDL)

\footnotetext{
* Correspondence: tkelesidis@mednet.ucla.edu

'Division of Infectious Diseases, Department of Medicine, David Geffen

School of Medicine, University of California, Los Angeles, CA, 90095, USA

Full list of author information is available at the end of the article
}

may play a role in this process; higher serum levels of pro-inflammatory HDL are associated with the increased rates of CVD in certain chronic inflammatory conditions such as rheumatoid arthritis, systemic lupus erythematosus, and type II diabetes mellitus [3]. While immune activation is a hallmark of HIV-1 infection, it is not known if HIV-1 infection affects levels of pro-inflammatory HDL, or if increases in HDL cholesterol (HDL-C) observed after initiation of ART in longitudinal studies are neutral, atheroprotective or atherogenic in nature $[2,4-8]$.

With regards to pro-inflammatory lipids, apoA-1 mimetic peptides have been shown to remove oxidation products from lipoproteins and cell membranes and restore structure and function of low density lipoprotein (LDL) and HDL in a wide range of experimental inflammatory conditions in animals [9]. In view of the systemic immune activation that is not entirely reversed despite effective ART $[9,10]$ and which could therefore be conducive to generating pro-inflammatory lipids, we hypothesized that HIV-1-infected persons on ART would have pro-inflammatory HDL, and that an apoA-1

\section{() Biomed Central}


mimetic peptide might reverse the inflammatory properties of HDL in these persons.

\section{Materials and methods Patients}

To test this hypothesis, plasma was obtained from 10 HIV-1-infected individuals on combination antiretroviral therapy with suppressed viremia (below 50 copies of $\mathrm{RNA} / \mathrm{ml}$ ). Informed consent was obtained from all subjects.

\section{Determination of proinflammatory HDL}

Plasma was incubated with the apoA-I mimetic peptide $\mathrm{L}-4 \mathrm{~F}$ at a concentration of $1 \mu \mathrm{g} / \mathrm{mL}$ for 15 minutes at $37^{\circ} \mathrm{C}$, or sham-treated prior to FPLC fractionation. HDL-containing FPLC fractions were tested for their ability to inhibit LDL-induced MCP-1 production in cultures of human aortic endothelial cells as previously described [9]. Results were normalized as a ratio to the effect of control LDL without HDL to yield the HDL inflammatory index (HII). More specifically HII is calculated from the ratio LDL-induced monocyte chemotactic activity (as determined by MCP-1 production in cultures of human aortic endothelial cells) in the presence of HDL/LDL-induced monocyte chemotactic activity in cultures of human aortic endothelial cells in the absence of HDL [9]. Data were analyzed with Student's t-test using SPSS 13.5 software (Chicago, IL, USA) and expressed as mean $\pm \mathrm{SD}$; P-values less than 0.05 were considered significant.

\section{Results}

All subjects were men with an average age of $43 \pm 14$ years ( \pm SD, range $21-63$ ) on combination ART. Viral load and $\mathrm{CD} 4^{+} \mathrm{T}$ cell counts were available on nine subjects (Table 1 ); these had plasma HIV-1 RNA values $<50$ copies/mL and mean blood CD4 ${ }^{+} \mathrm{T}$ cell counts of $595 \pm 166$ cells $/ \mathrm{mm}^{3}$ (range 367 - 966). Mean HII values for HDL in the sham treated plasma were $2.16 \pm 0.63$ (range 1.01 - 2.73) while mean HII values for HDL in the L-4F treated plasma were significantly lower $(\mathrm{p}<0.0001)$ at $0.89 \pm 0.16$ (range 0.64 1.19). These values were compared to previously reported mean values from healthy controls $(0.43 \pm 0.05$ and $0.20 \pm$ 0.04 for sham and 4F treatment respectively (Figure 1) [9]. Thus, high-density lipoprotein isolated from plasma of persons with ART treated HIV-1 infection was highly proinflammatory, and was markedly less pro-inflammatory after treatment of plasma with $4 \mathrm{~F}$ in vitro (Figure 1). Both the high baseline HII and reduction of the inflammatory index after $4 \mathrm{~F}$ treatment were observed in all 10 samples, suggesting an overall pro-inflammatory lipid profile that was at least partially reversible by $4 \mathrm{~F}$.

\section{Discussion}

Classically, HDL is believed to play an important role in mitigating oxidative stress and inflammation by uptake, processing and disposal of oxidized lipids [2,11]. However, systemic inflammation has been shown to lower this antioxidant and anti-inflammatory activity by transforming HDL to a pro-oxidant, pro-inflammatory acutephase HDL that enhances the tendency of LDL to induce monocyte chemotaxis [2]. It has been demonstrated that mice that are genetically susceptible to develop atherosclerosis have augmented pro-inflammatory HDL $[9,11]$. HDL, therefore, not only removes excess LDL-derived cholesterol from peripheral tissues, but also has a major role in mitigating LDL-induced inflammation.

In our small cohort, all HIV-1-infected persons showed a marked reduction of HDL anti-inflammatory activity as evidenced by impaired HDL mediated inhibition of LDLinduced monocyte chemotactic activity. The observed defect cannot be attributed to low plasma HDL level in

Table 1 Characteristics of the HIV-1-infected study participants

\begin{tabular}{|c|c|c|c|c|c|c|c|c|c|c|c|}
\hline Subject & Age & Sex & $\begin{array}{l}\text { CD4 count } \\
\text { (cells } / \mathrm{mm}^{3} \text { ) }\end{array}$ & $\mathrm{VL}(\mathrm{RNA} / \mathrm{ml})$ & $\mathrm{TC}(\mathrm{mg} / \mathrm{dl})$ & $\mathrm{HDL}(\mathrm{mg} / \mathrm{dl})$ & $\mathrm{LDL}(\mathrm{mg} / \mathrm{dl})$ & TG (mg/dl) & HII (sham) & HII (L-4F) & ART \\
\hline 1 & 25 & $M$ & 367 & $<50$ & 135 & 51 & 66 & 91 & 2.45 & 0.75 & $\overline{A Z V, ~ R T V, ~ T D F, ~ F T C ~}$ \\
\hline 2 & NA & M & NA & NA & NA & NA & NA & NA & 2.69 & 1.19 & NA \\
\hline 3 & 57 & M & 499 & $<50$ & 159 & 31 & 77 & 254 & 1.01 & 0.69 & $E F V, A B C, 3 T C$ \\
\hline 4 & 44 & M & 540 & $<50$ & NA & NA & NA & NA & 2.73 & 0.96 & TDF, FTC, RTV, FPV \\
\hline 5 & 44 & M & 540 & $<50$ & 286 & 26 & 212 & 240 & 2.55 & 0.93 & TDF, FTC, AZV, RTV \\
\hline 6 & 46 & M & 571 & $<50$ & 184 & 52 & 108 & 121 & 1.82 & 0.89 & NA \\
\hline 7 & 47 & M & 966 & $<50$ & 176 & 52 & 122 & 100 & 2.61 & 0.86 & TDF, FTC, NVP \\
\hline 8 & 31 & M & 553 & $<50$ & 205 & $76^{*}$ & 109 & 98 & 1.24 & 0.64 & $\mathrm{EFV}, \mathrm{FTC}, \mathrm{TDF}$ \\
\hline 9 & 21 & M & 712 & $<50$ & 169 & $64^{*}$ & 93 & 58 & 1.95 & 1.00 & EFV, FTC, TDF \\
\hline 10 & 62 & M & 605 & $<50$ & 167 & 44 & 88 & 176 & 2.57 & 1.00 & LPV, RTV, 3TC ETV \\
\hline
\end{tabular}

Abbreviations: AZV: Atazanavir; ABC: Abacavir; ART: Antiretroviral therapy; ETV: Etravirine; Efavirenz (EFV), FTC: Emtricitabine, FPV: Fosamprenavir; HDL: High Density Lipoprotein; HII; HDL Inflammatory Index; 3TC: Lamivudine; LPV: lopinavir; LDL: Low Density Lipoprotein; M: Male; NVP: Nevirapine; NA: Not available; TDF: Tenofovir; TC: Total Cholesterol; TG: Triglycerides; RTV: Ritonavir; VL: Viral Load

*:Samples were collected when patients were non-fasting. 


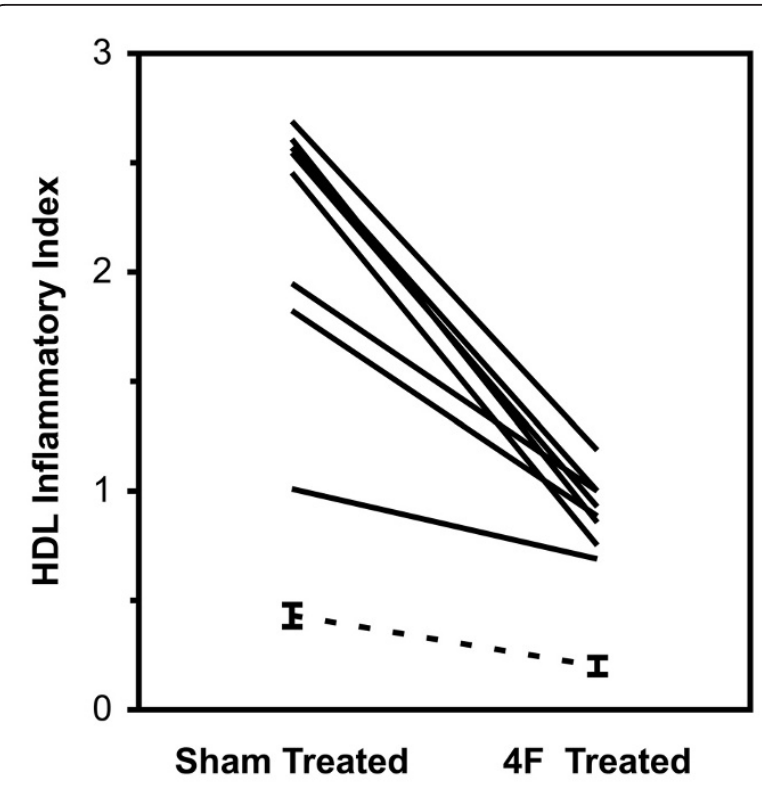

Figure $1 \mathrm{HDL}$ inflammatory index from plasma of patients with HIV (sham-treated versus L-4F- treated). The dotted line indicates previously reported values from healthy controls $(0.43 \pm 0.05$ and $0.20 \pm 0.04$ for sham and $4 \mathrm{~F}$ treatment respectively, taken from reference 4). In this assay, anti-inflammatory $\mathrm{HDL}$ is defined as $\mathrm{HDL}$ inflammatory index values $<1.0$ and proinflammatory $\mathrm{HDL}$ is defined as HDL inflammatory index values $>1.0$.

these patients, as equal amounts of isolated HDL were assayed; thus the observed HDL dysfunction must be due primarily to qualitative abnormalities. Oxidative stress and inflammation are nearly constant features of HIV-1 infection and the prevailing inflammatory state likely contributes to the observed reduction of HDL antiinflammatory function in this population. This could lead to a vicious cycle in which the underlying inflammation and oxidative stress induce HDL dysfunction, which results in further inflammation.

Treatment of plasma samples from the HIV-1-infected subjects with the potent apoA-1 mimetic peptide $4 \mathrm{~F}$ results in a marked increase in HDL anti-inflammatory activity. $4 \mathrm{~F}$ is an 18-amino acid apoA-1 mimetic peptide, and its anti-inflammatory effects are mediated by its ability to preferentially remove oxidation products from lipoproteins and cell membranes, thereby restoring HDL and LDL function and structure, improving cell function, and attenuating inflammation [11]. ApoA-1 mimetic peptides have been shown to reduce atherosclerosis and attenuate inflammation in experimental animals without significantly changing plasma lipid levels [11]. 4F treatment in this study generally failed to return the pro-inflammatory activity of HDL to the low levels that we have previously reported in healthy controls [9].

These preliminary observations suggest that the anti-inflammatory properties of HDL are defective in
HIV-1-infected persons despite treatment that is considered to be virologically successful, and that standard clinical lipid profile testing may not be an adequate measurement of the risk for CVD in these individuals. In this small pilot study we did not assess the possible association of proinflammatory HDL with other markers of immune activation and inflammation and the possible effect of $4 \mathrm{~F}$ on these markers. An ongoing prospective study will aim at addressing the possible association of proinflammatory HDL with other markers of inflammation, immune activation and progression of atherosclerosis in patients with HIV infection. This study will also evaluate the effect of different types of antiretroviral treatment regimens versus HIV infection itself on the HDL inflammatory index.

In conclusion, to our knowledge this is the first demonstration that HIV-1 infection is associated with a marked reduction of HDL antioxidant/anti-inflammatory activities. In vitro, these abnormalities were significantly improved by treatment with the apoA-1 mimetic peptide, 4F.

\section{Abbreviations used}

ART: antiretroviral therapy; apoA-1: apolipoprotein A-1; CVD: cardiovascular disease; FPLC: Fast protein liquid chromatography; HDL: High Density Lipoprotein; HIV-1: Human Immunodeficiency virus-1; HII: HDL inflammatory index; LDL: Low Density Lipoprotein; MCP-1: Monocyte chemotactic protein1

\section{Acknowledgements}

This work was supported by US Public Health Service grants HL095132 (JSC), HL-30568 (AMF and MN) and the Laubisch, Castera, and M.K. Grey Funds at UCLA.

Disclosures

AMF and MN are principals in Bruin Pharma and AMF is an officer in Bruin Pharma. This work was presented at the 11th International Workshop on Adverse Drug Reactions and Co-morbidities in HIV (26-28 October 2009, Philadelphia, PA, USA, abstract no. P-31)

\section{Author details}

'Division of Infectious Diseases, Department of Medicine, David Geffen School of Medicine, University of California, Los Angeles, CA, 90095, USA. ${ }^{2}$ Department of Microbiology, Immunology, and Molecular Genetics, David Geffen School of Medicine, University of California, Los Angeles, CA, 90095, USA. ${ }^{3}$ Division of Cardiology, Department of Medicine, David Geffen School of Medicine, University of California, Los Angeles, CA, 90095, USA.

\section{Authors' contributions}

All authors contributed to the intellectual development of this work, and approved the final manuscript. TK, OY, KN and MN analyzed the data. TK searched the literature and wrote the draft paper. JC and AFM provided critical corrections to the manuscript.

\section{Competing interests}

AMF and MN are principals in Bruin Pharma and AMF is an officer in Bruin Pharma.

Received: 5 January 2011 Accepted: 23 February 2011

Published: 23 February 2011

\section{References}

1. Currier JS: Update on cardiovascular complications in HIV infection. Top HIV Med 2009, 17:98-103. 
2. Barter PJ, Nicholls S, Rye KA, Anantharamaiah GM, Navab M, Fogelman AM: Antiinflammatory properties of HDL. Circ Res 2004, 17:764-772.

3. McMahon M, Grossman J, FitzGerald J, et al: Proinflammatory high-density lipoprotein as a biomarker for atherosclerosis in patients with systemic lupus erythematosus and rheumatoid arthritis. Arthritis Rheum 2006, 54:2541-2549.

4. Buchacz K, Weidle PJ, Moore D, et al: Changes in lipid profile over 24 months among adults on first-line highly active antiretroviral therapy in the home-based AIDS care program in rural Uganda. J Acquir Immune Defic Syndr 2008, 47:304-311.

5. Hsue PY, Hunt PW, Sinclair E, et al: Increased carotid intima-media thickness in HIV patients is associated with increased cytomegalovirusspecific T-cell responses. AIDS 2006, 20:2275-2283.

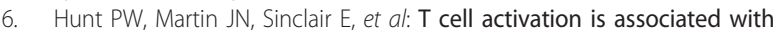
lower CD4+ T cell gains in human immunodeficiency virus-infected patients with sustained viral suppression during antiretroviral therapy. J Infect Dis 2003, 187:1534-1543.

7. Baker JV, Neuhaus J, Duprez D, et al: Changes in Inflammatory and Coagulation Biomarkers: A Randomized Comparison of Immediate versus Deferred Antiretroviral Therapy in Patients With HIV Infection. J Acquir Immune Defic Syndr 2011, 56:36-43.

8. Kuller LH, Tracy R, Belloso W, et al: Inflammatory and coagulation biomarkers and mortality in patients with HIV infection. PLOS Med 2008, 5:e203.

9. Vaziri ND, Moradi H, Pahl MV, Fogelman AM, Navab M: In vitro stimulation of HDL anti-inflammatory activity and inhibition of LDL proinflammatory activity in the plasma of patients with end-stage renal disease by an apoA-1 mimetic peptide. Kidney Int 2009, 76:437-444.

10. Almeida CA, Price $P$, French MA: Immune activation in patients infected with HIV type 1 and maintaining suppression of viral replication by highly active antiretroviral therapy. AIDS Res Hum Retroviruses 2002, 18:1351-1355.

11. Navab M, Shechter I, Anantharamaiah GM, Reddy ST, Van Lenten BJ, Fogelman AM: Structure and function of $\mathrm{HDL}$ mimetics. Arterioscler Thromb Vasc Biol 2010, 30:164-168.

doi:10.1186/1476-511X-10-35

Cite this article as: Kelesidis et al: HIV-1 infected patients with suppressed plasma viremia on treatment have pro-inflammatory HDL. Lipids in Health and Disease 2011 10:35.

\section{Submit your next manuscript to BioMed Central and take full advantage of:}

- Convenient online submission

- Thorough peer review

- No space constraints or color figure charges

- Immediate publication on acceptance

- Inclusion in PubMed, CAS, Scopus and Google Scholar

- Research which is freely available for redistribution

Submit your manuscript at www.biomedcentral.com/submit
Biomed Central 